\title{
$O-\left(2-\left[{ }^{18} \mathrm{~F}\right]\right.$ fluoroethyl)-L-tyrosine PET in gliomas: influence of data processing in different centres
}

\author{
Christian P. Filss ${ }^{1,2}$, Nathalie L. Albert ${ }^{3}$, Guido Böning ${ }^{3}$, Elena Rota Kops ${ }^{1}$, Bogdana Suchorska ${ }^{4}$, Gabriele Stoffels ${ }^{1}$, \\ Norbert Galldiks ${ }^{1,5,6}$, Nadim J. Shah ${ }^{1,7,8}$, Felix M. Mottaghy ${ }^{2,8}$, Peter Bartenstein ${ }^{3}$, Jörg C. Tonn ${ }^{4}$ \\ and Karl-Josef Langen ${ }^{1,2,8^{*}}$
}

\begin{abstract}
Background: PET using O-(2-[18 F]fluoroethyl)-L-tyrosine $\left({ }^{18} \mathrm{~F}-\mathrm{FET}\right)$ is an established method for brain tumour diagnostics, but data processing varies in different centres. This study analyses the influence of methodological differences between two centres for tumour characterization with ${ }^{18}$ F-FET PET using the same PET scanner. Methodological differences between centres A and B in the evaluation of ${ }^{18} \mathrm{~F}$-FET PET data were identified for (1) framing of PET dynamic data, (2) data reconstruction, (3) cut-off values for tumour delineation to determine tumour-to-brain ratios (TBR) and tumour volume $\left(T_{\text {vol }}\right)$ and (4) ROI definition to determine time activity curves (TACs) in the tumour. Based on the ${ }^{18}$ F-FET PET data of 40 patients with untreated cerebral gliomas (20 WHO grade II, 10 WHO grade III, 10 WHO grade IV), the effect of different data processing in the two centres on $T_{B R}$ mean, $T B R_{\text {max }}, T_{\text {vol, }}$ time-to-peak (TTP) and slope of the TAC was compared. Further, the effect on tumour grading was evaluated by ROC analysis.

Results: Significant differences between centres A and B were found especially for TBR $\max (2.84 \pm 0.99$ versus $3.34 \pm 1.13 ; p<0.001), T_{\text {vol }}(1.14 \pm 1.28$ versus $1.51 \pm 1.44 ; p<0.001)$ and $T \mathrm{TP}(22.4 \pm 8.3 \mathrm{~min}$ versus $30.8 \pm 6.3 \mathrm{~min} ; p<0.001)$ and minor differences for TBR mean and slope. Tumour grading was not influenced by different data processing.

Conclusions: Variable data processing of ${ }^{18} \mathrm{~F}$-FET PET in different centres leads to significant differences especially for $\mathrm{TBR}_{\max }$ and $\mathrm{T}_{\mathrm{vol}}$. A standardization of data processing and evaluation is needed to make ${ }^{18} \mathrm{~F}$-FET PET comparable between different centres.
\end{abstract}

Keywords: Brain tumours, FET PET, Tumour-to-brain ratios, Dynamic FET PET

\section{Background}

PET using the amino acid $O-\left(2-{ }^{18} \mathrm{~F}\right.$-fluoroethyl $)$-L-tyrosine $\left({ }^{18} \mathrm{~F}-\mathrm{FET}\right)$ has received increasing attention for brain tumour diagnostics due to logistic advantages of ${ }^{18} \mathrm{~F}$ labelling (half-life, $109.8 \mathrm{~min}$ ) compared with $\mathrm{L}$-[methyl- $\left.{ }^{11} \mathrm{C}\right]$-methionine $\left({ }^{11} \mathrm{C}-\mathrm{MET}\right)$ PET, efficient radiosynthesis and high in-vivo stability [1-6]. Multiple studies have demonstrated the clinical potential of ${ }^{18} \mathrm{~F}$ -

\footnotetext{
* Correspondence: k.j.langen@fz-juelich.de

${ }^{1}$ Institute of Neuroscience and Medicine (INM-4, INM-3), Forschungszentrum Jülich, Jülich, Germany

${ }^{2}$ Department of Nuclear Medicine, RWTH University of Aachen, Aachen, Germany

Full list of author information is available at the end of the article
}

FET PET to determine the extent of cerebral gliomas for biopsy guidance, treatment planning, detection of tumour recurrence, estimation of prognosis in newly diagnosed and untreated gliomas and treatment monitoring [7-12]. ${ }^{18} \mathrm{~F}$-FET uptake in the tumour is usually expressed by mean and maximum tumour-to-brain ratios $\left(\mathrm{TBR}_{\text {mean }}, \mathrm{TBR}_{\max }\right)$ within a scan period between 20 and $40 \mathrm{~min}$ after injection. Furthermore, the timeactivity curves (TACs) of ${ }^{18} \mathrm{~F}$-FET uptake typically show differences in high-grade and low-grade gliomas or nonneoplastic lesions which provide valuable additional information for tumour grading or differential diagnosis [13-15]. Thus, continuously increasing ${ }^{18}$ F-FET uptake 
is more frequently observed in low-grade gliomas and nonneoplastic lesions, while kinetics with an early peak of ${ }^{18} \mathrm{~F}$-FET uptake in the first $10-20 \mathrm{~min}$ after injection followed by a decreasing TAC is a common finding in more aggressive tumours like high-grade glioma or brain metastases $[10,16,17]$. TBR and dynamic parameters of ${ }^{18} \mathrm{~F}$-FET uptake may be influenced on the one hand by the spatial resolution of the PET scans which is dependent on the scanner type, reconstruction algorithms and data filtering and on the other hand by the definition of the region of interest (ROI) in the tumour and the brain. There is some controversy about the diagnostic accuracy of TAC analysis for tumour grading raising the question whether discrepant results in different centres may be caused by differences in the methodology, patient population or other reasons such as neuropathological interpretation.

In the present study, methodological differences between two large centres in Germany (A = Ludwig-MaximiliansUniversity Munich, Germany; B = Forschungszentrum Jülich, Germany) with a high number of ${ }^{18}$ F-FET PET investigations (>500/year) and multiple publications in the field were identified. Based on a balanced group of $20 \mathrm{pa}-$ tients with high-grade glioma of WHO grade III and IV (HGG) and 20 patients with low-grade glioma of WHO grade II (LGG) [18] who were investigated with the same scanner used in both centres, the influence of methodological differences between the two centres on common parameters for tumour characterization with ${ }^{18} \mathrm{~F}$-FET PET was evaluated. This analysis may be helpful to develop a more standardized approach in order to make the data between different centres more comparable.

\section{Methods}

\section{Patient population}

The data of 40 adults, previously untreated patients with brain tumours who were investigated with ${ }^{18} \mathrm{~F}$-FET PET at the Forschungszentrum Jülich between June 2006 and April 2014, were selected randomly from our data base and included in this study. Histopathological diagnosis was available for all patients obtained either by biopsy or tumour resection which was performed within 3 months after ${ }^{18}$ F-FET PET. Diagnosis was astrocytoma WHO grade II in 20 cases, anaplastic astrocytoma WHO grade III in 10 cases and glioblastoma WHO grade IV in 10 cases according to Louis et al. [18]. All patients were investigated within a prospective study evaluating the diagnostic value ${ }^{18}$ F-FET PET in cerebral gliomas which was approved by the university ethics committee and federal authorities (study no. 2438, University of Düsseldorf). All subjects gave prior written informed consent for their participation in the ${ }^{18} \mathrm{~F}$-FET PET study and evaluation of their data for scientific purposes.

\section{${ }^{18}$ F-FET PET}

The amino acid ${ }^{18}$ F-FET was produced via aminopolyether-activated nucleophilic ${ }^{18} \mathrm{~F}$-fluorination and applied as described previously [19]. Dynamic PET data were acquired in list mode for $40 \mathrm{~min}$ after intravenous injection of approx. $200 \mathrm{MBq}{ }^{18}$ F-FET. The measurements were performed on an ECAT Exact $\mathrm{HR}+$ scanner in 3D mode (Siemens Medical Systems, Knoxville, TN, USA: 32 rings, axial field of view $15.5 \mathrm{~cm}$, image resolution $5.5 \mathrm{~mm}$ ) which is routinely used for ${ }^{18}$ F-FET PET in both centres. For attenuation correction, transmission scans with three ${ }^{68} \mathrm{Ge} /{ }^{68} \mathrm{Ga}$ rotating line sources were measured. Raw PET data were corrected for random and scattered coincidences as well as dead time.

\section{Data processing}

Methodological differences between centres A and B were identified for the framing of dynamic PET data and data reconstruction (Table 1). Therefore, the PET data were processed in two different ways according to the individual approach established in centre A and centre B. For centre $\mathrm{A}$, the dynamic data set was framed into time intervals of $6 \times 10 \mathrm{~s}, 4 \times 30 \mathrm{~s}, 1 \times 2 \mathrm{~min}, 3 \times 5 \mathrm{~min}$ and $2 \times 10 \mathrm{~min}$ and the data were reconstructed by filtered back projection (FBP) using a 5-mm Hann filter. For centre $\mathrm{B}$, the framing was $5 \times 1 \mathrm{~min}, 5 \times 3 \mathrm{~min}$ and $4 \times 5 \mathrm{~min}$ followed by iterative reconstruction (ITR) (ordered-subset expectation maximization, 6 iterations, 16 subsets). ${ }^{18}$ F-FET uptake in the tissue was expressed as standardized uptake value (SUV) by dividing the radioactivity $(\mathrm{kBq} / \mathrm{ml})$ in the tissue by the radioactivity injected per gram of body weight. An example of a brain tumour for the time interval from 20 to 40 min after injection for

Table 1 Comparison of methodology centre A and centre B

\begin{tabular}{lll}
\hline & Centre A & Centre B \\
\hline Scanner & LMU & FZJ \\
Framing & ECAT Exact HR+ PET & ECAT Exact HR+ PET \\
Reconstruction & $6 \times 10^{\prime \prime}, 4 \times 30^{\prime \prime}, 1 \times 2^{\prime}, 3 \times 5^{\prime}, 2 \times 10^{\prime}$ & $5 \times 1^{\prime}, 5 \times 3^{\prime}, 6 \times 5^{\prime}$ \\
Definition of tumour volume (cut-off) & FBP $(5-\mathrm{mm}$ Hann filter) & Iterative $6 \mathrm{i} / 16 \mathrm{~s}$ \\
ROI for TAC definition & TBR $>1.8$ & TBR $>1.6$ \\
\hline
\end{tabular}


the different data processing in centres $\mathrm{A}$ and $\mathrm{B}$ is shown in Fig. 1. Corresponding evaluation of a Jaszczak phantom with the two methods is shown in Fig. 2.

\section{Definition of tumour ROI}

Further differences between the two centres were identified for the definition of the region of interest (ROI) for the tumour which was used to determine the tumour volume $\left(\mathrm{T}_{\mathrm{vol}}\right)$ and the $\mathrm{TBR}_{\max }$ and $\mathrm{TBR}_{\text {mean }}$. For the two data sets generated by the different data processing in centres $\mathrm{A}$ and $\mathrm{B}$, the definition of these tumour ROIs was based on the summed images of ${ }^{18} \mathrm{~F}$-FET uptake from 20 to $40 \mathrm{~min}$ after injection in the slice with the maximal ${ }^{18}$ F-FET uptake in the tumour. The background ROI was placed in the contralateral hemisphere to the tumour in an area of normal-appearing brain tissue including white and grey matter, identically for centres A and B. For the data set of centre A, the tumour volume $\left(\mathrm{T}_{\mathrm{vol}}\right)$ was delineated using a cut-off of the TBR $>1.8$ which is based on the clinical experience in that centre $[16,20]$. For the data set of centre $B, T_{v o l}$ was delineated using a cut-off of the TBR $>1.6$ based on a previous biopsy controlled study [8]. $\mathrm{TBR}_{\max }$ and $\mathrm{TBR}_{\text {mean }}$ were calculated by dividing the mean and maximum SUV in the tumour ROI by the mean SUV of the background region in the normal brain tissue. An example is shown in Fig. 3.

For analysis of dynamic PET data, all TACs were corrected for radioactive decay of ${ }^{18} \mathrm{~F}$. Centre $\mathrm{B}$ used a single tumour ROI which was placed in the slice with maximal tracer uptake in the 20-40-min summation images and which was defined by the cut-off TBR $>1.6$ as described above. In centre A, multiple slices of the tumour area were screened and a 90\% isocontour ROI of the local tumour maxima was created in the individual slices of the summation image 10-30 min p.i. and TACs were recorded for each slice of the tumour region (Fig. $3 \mathrm{~b})$. The TAC with the most negative slope was identified, and the most negative slope being present in at least two adjacent slices was selected for further TAC evaluation. In order to compare the TACs produced by the two different centres quantitatively, the time-to-peak (TTP; time in minutes from the beginning of the dynamic acquisition up to the maximum SUV of the lesion) was determined based on different ROIs determined for centres A and B. Furthermore, we quantified the slope of the TAC in the late phase of ${ }^{18} \mathrm{~F}$-FET uptake by fitting a linear regression line to the late phase of the curve (10-40 min p.i.). The slope was expressed in change of SUV per hour.

\section{Statistical analysis}

Descriptive statistics are provided as mean and standard deviation. To compare the results obtained with the different approaches in the two centres based on the same raw data, the paired $t$ test and Pearson's correlation coefficient was used. The signed rank test was used when variables were not distributed normally. $P<0.05$ was considered significant. The diagnostic performance of the different parameters to differentiate between LGG (WHO grade II) and HGG (WHO grade III and IV) was assessed by analyses of receiver operating characteristic (ROC) curves using the histological confirmation as reference. The area under the ROC curve (AUC) was used to compare the results obtained with different methodologies. Statistical analysis was performed using SigmaPlot software (SigmaPlot v11.0, Systat Software). No correction for multiple testing was included.

\section{Results}

\section{Static PET data}

$\mathrm{TBR}_{\max }$ values as determined by the different methodologies of centres A and B showed a significant correlation $(r=0.98, p<0.001)$, but there was a deviation from the line of unity towards higher $\mathrm{TBR}_{\max }$ values for centre B (Fig. 4). The mean values of $\mathrm{TBR}_{\max }$ in centre A were significantly lower than that in centre $\mathrm{B}$ (A/ $\mathrm{TBR}_{\max } 2.84 \pm 0.99$ versus $\mathrm{B} / \mathrm{TBR}_{\max } 3.34 \pm 1.13$,

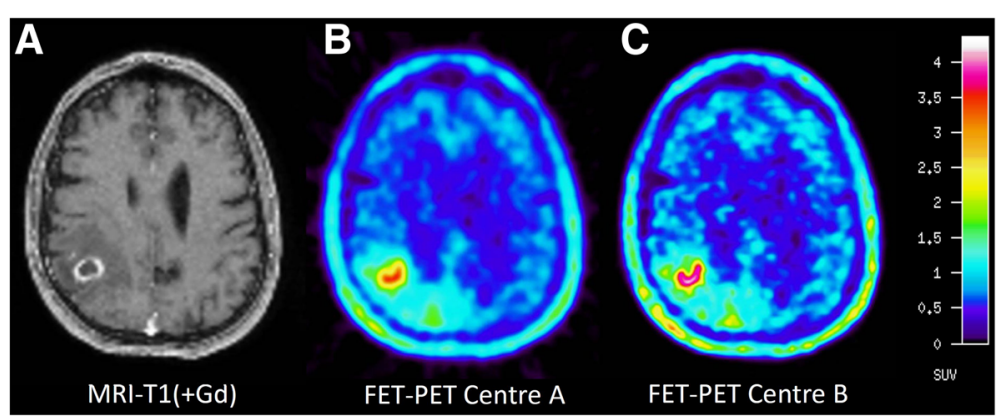

Fig. 1 Glioblastoma in the right parietal lobe. Contrast-enhanced T1-weighted MRI (a) shows a ring-enhancing lesion. FET PET image based on the method of centre A (b) shows lower noise but the image based on the method of centre B (c) shows a sharper demarcation of the metabolically active tumour parts 

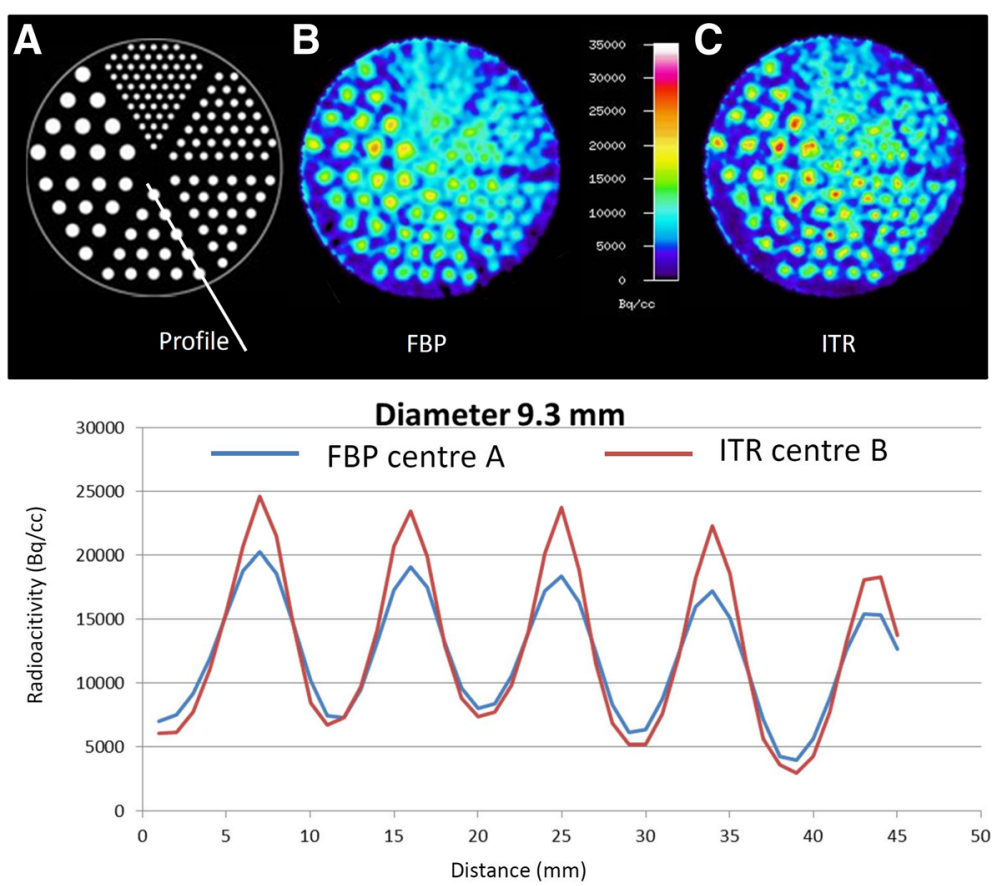

Fig. 2 Jasczack phantom (a) with tubes of different size filled with radioactivity and reconstructed according to the procedure in centre $\mathbf{A}(\mathbf{b}$, blue profile line) and centre $\mathbf{B}$ (c, red profile line). The method of centre $\mathbf{A}$ shows about 20\% lower maximum values in the tubes with a diameter of $9.3 \mathrm{~mm}$ which is mainly due to reconstruction by filtered back projection in centre $\mathbf{A}$ instead of iterative reconstruction (centre $\mathbf{B}$ )

$p<0.001$ ) (Table 2). This is also reflected by the lower peak values in the profile of count rates across the tubes with a diameter of $9.3 \mathrm{~mm}$ in the Jaszczak phantom (Fig. 2).

ROC analysis yielded a similar AUC for the differentiation of high-grade and low-grade gliomas using the $\mathrm{TBR}_{\max }$ values determined with the different approaches (AUC $\mathrm{TBR}_{\max }$ centre $\mathrm{A}=0.77$ and $\mathrm{AUC} \mathrm{TBR}_{\max }$ centre $\mathrm{B}=0.78)$ (Table 2).

$\mathrm{TBR}_{\text {mean }}$ values as determined by the different methodologies of centres A and B showed a significant correlation $(r=0.95, p<0.001)$, which was nearly congruent with the line of unity (Fig. 5). The mean values of
$\mathrm{TBR}_{\text {mean }}$ were nearly identical although a weak significance was noted in the paired $t$ test $\left(\mathrm{A} / \mathrm{TBR}_{\text {mean }}\right.$ $2.20 \pm 0.41$ versus $\mathrm{B} / \mathrm{TBR}_{\text {mean }} 2.16 \pm 0.41, p<0.04$ ) (Table 2). ROC analysis yielded a similar AUC for the differentiation of high-grade and low-grade gliomas using the $\mathrm{TBR}_{\text {mean }}$ values determined with the different approaches (AUC $\mathrm{TBR}_{\text {mean }}$ centre $\mathrm{A}=0.78$ and $\mathrm{AUC}$ $\mathrm{TBR}_{\text {mean }}$ centre $\mathrm{B}=0.76$ ) (Table 2 ).

$\mathrm{T}_{\mathrm{vol}}$ values calculated according to the different methodologies of centres A and centre B showed a significant correlation $(r=0.99, p<0.001)$, but there was a significant difference of the mean values $\left(\mathrm{T}_{\mathrm{vol} \mathrm{A}} 1.14 \mathrm{ml} \pm 1.28 \mathrm{ml}\right.$

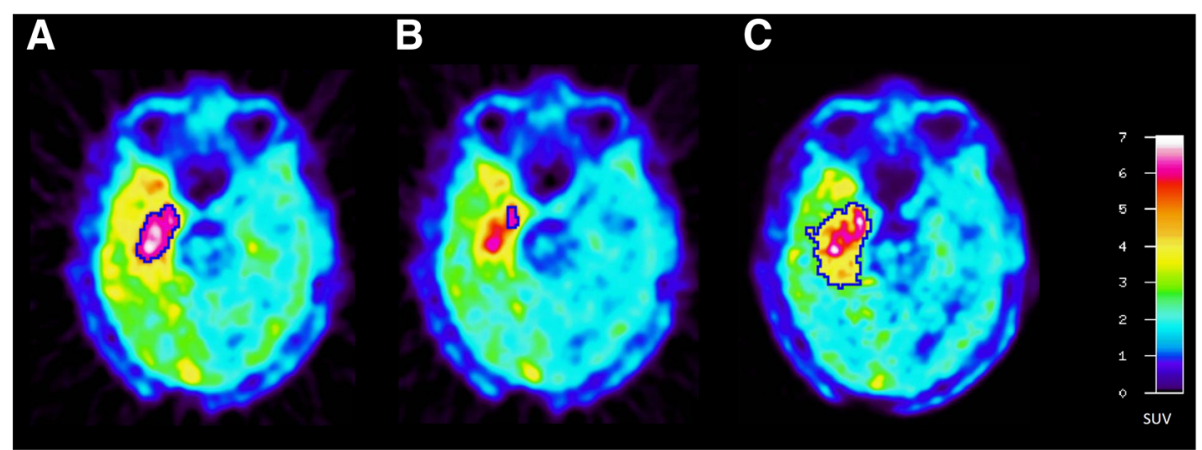

Fig. $3 \mathrm{ROI}$ definition in a patient with a glioblastoma in the right temporal lobe. $\mathbf{a}$ The tumour volume as delineated by TBR $>1.8$ based on data reconstruction of centre $\mathbf{A}$. b The $90 \%$ isocontour of the 10-30-min image for TAC generation in centre $\mathbf{A}$ and $\mathbf{c}$ the tumour volume as delineated by a TBR $>1.6$ in centre $B$ which is also used TAC generation 


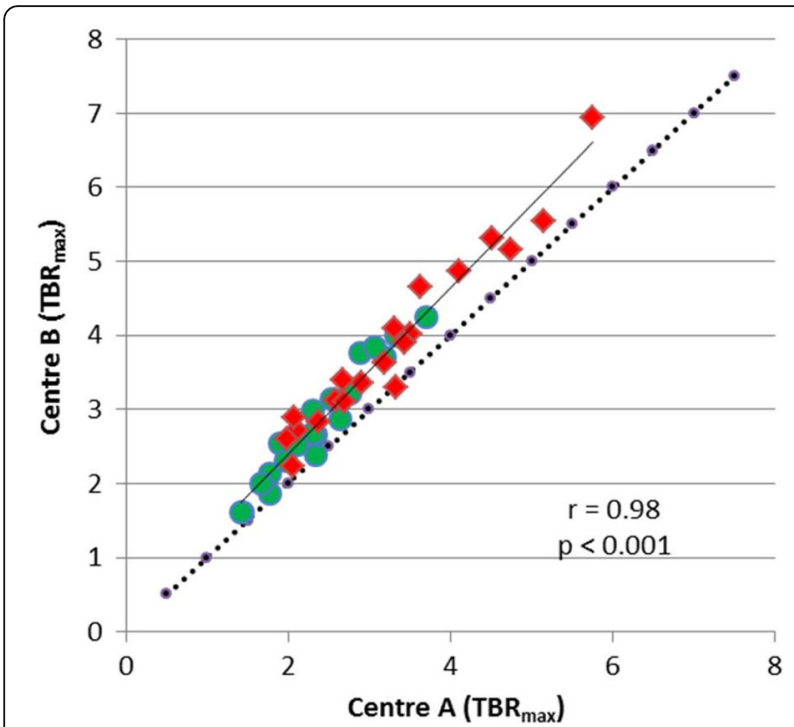

Fig. $\mathbf{4}$ Correlation of $\mathrm{TBR}_{\max }$ from centres $\mathbf{A}$ and $\mathbf{B}$. There is a significant correlation but a deviation from line of unity indicating higher $\mathrm{TBR}_{\max }$ values for centre $\mathbf{B}$. High-grade tumours are indicated by red symbols and low-grade tumour by green symbols

versus $\mathrm{T}_{\text {vol в }} 1.51 \mathrm{ml} \pm 1.43 \mathrm{ml}$ ), which leads to relevant differences in the definition of the "biological tumour volume".

\section{Dynamic PET data}

TTP values as determined by the different methodologies of centres $\mathrm{A}$ and $\mathrm{B}$ showed a significant correlation $(r=0.61, p<0.001)$, but the correlation coefficient was considerably smaller than for the parameters mentioned above. There was a significant difference of the mean

Table 2 Comparison of ${ }^{18} \mathrm{~F}$-FET parameters in centre $\mathrm{A}$ and centre $\mathrm{B}$ based on the same data set

\begin{tabular}{lccl}
\hline & Centre A & Centre B & $p$ value \\
\hline Comparison of mean values of FET PET & parameters & \\
TBR $_{\text {max }}$ & $2.84 \pm 0.99$ & $3.34 \pm 1.13$ & $<0.001$ \\
TBR $_{\text {mean }}$ & $2.20 \pm 0.41$ & $2.16 \pm 0.41$ & $<0.04$ \\
$T_{\text {vol }}(\mathrm{ml})$ & $1.14 \pm 1.28$ & $1.51 \pm 1.44$ & $<0.001$ \\
TTP $(\mathrm{min})$ & $22.4 \pm 8.3$ & $30.8 \pm 6.3$ & $<0.001$ \\
Slope $(\mathrm{SUV} / \mathrm{h})$ & $-0.06 \pm 0.92$ & $0.73 \pm 0.69$ & $<0.001$
\end{tabular}

Comparison of accuracy in differentiating HG and LG gliomas (AUC in ROC analysis)

\begin{tabular}{llll}
$T B R_{\max }$ & 0.77 & 0.78 & n.s. \\
$\mathrm{TBR}_{\text {mean }}$ & 0.78 & 0.76 & n.s. \\
$T_{\text {vol }}$ & 0.78 & 0.76 & n.s. \\
$T T P$ & 0.73 & 0.78 & n.s. \\
Slope & 0.76 & 0.72 & n.s. \\
\hline
\end{tabular}

n.s. = not significant

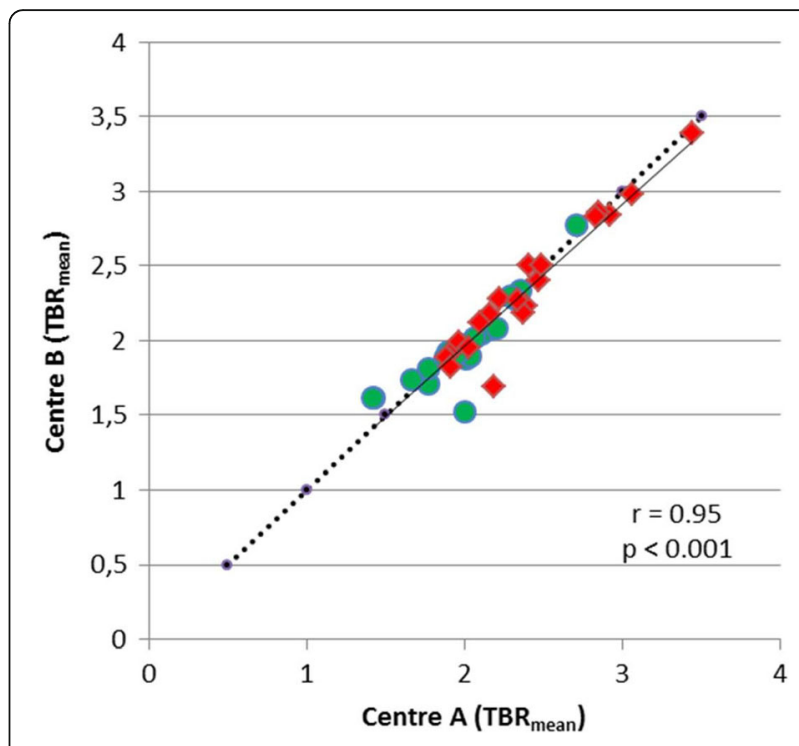

Fig. $\mathbf{5}$ Correlation of TBR mean from centres $\mathbf{A}$ and $\mathbf{B}$. There is a significant correlation which is close to the line of unity. High-grade tumours are indicated by red symbols and low-grade tumour by green symbols

values (TTP A $22.4 \quad \pm \quad 8.3 \quad \mathrm{~min}$ versus $\quad$ TTP $\mathrm{B}$ $30.8 \pm 6.3$ min, $p<0.001$ ).

Slope values calculated according to methodology of centres A and B showed a significant correlation $(r=0.90$, $p<0.001$ ) (Fig. 6). There was a significant difference of the mean values (slope A $-0.06 \pm 0.92 \mathrm{SUV} / \mathrm{h}$ versus slope B $0.73 \pm 0.69 \mathrm{SUV} / \mathrm{h})$. For both TTP and slope, ROC

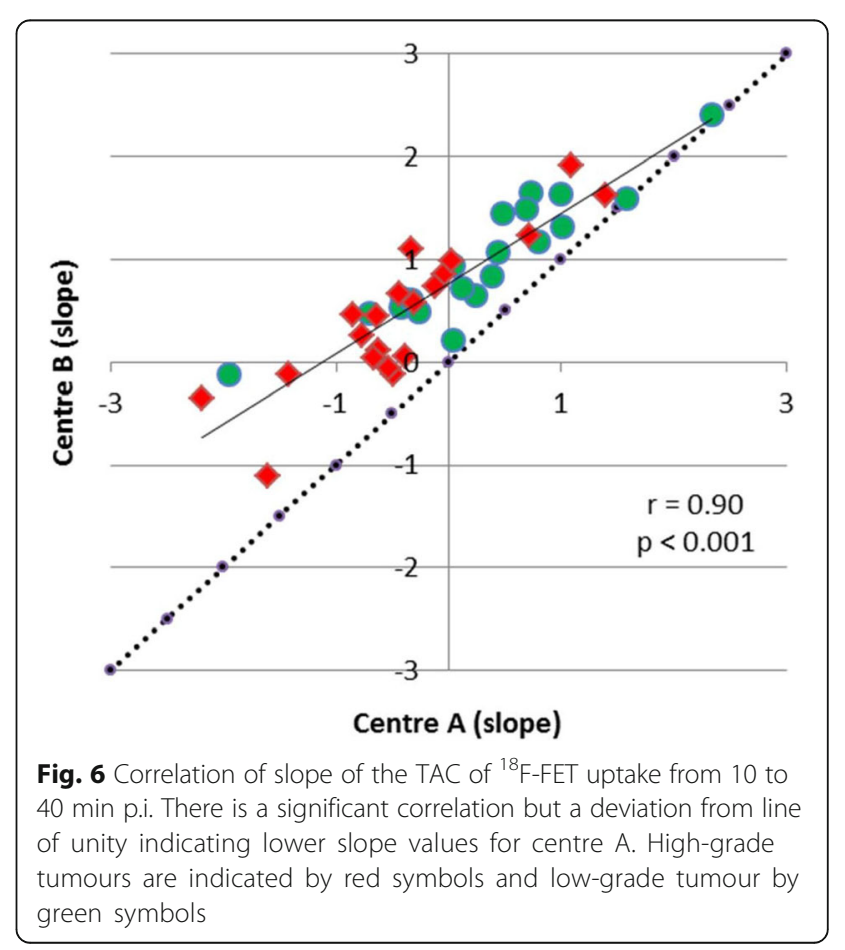


analysis yielded a similar AUC for the differentiation between HGG and LGG determined with the two different approaches (Table 2). Comparison of the slope values in both centres with framing as the only variable using the same reconstruction method (FBP) and ROI size ( $1.6 \mathrm{~cm}$ ) for both data sets showed a correlation close to the line of unity (Fig. 7). Thus, an influence of different data framing in centres A and B could be excluded.

\section{Discussion}

PET using radiolabelled amino acids is gaining increasing interest for the diagnostics of brain tumours because conventional MRI is limited in differentiating tumour tissue from nonspecific tissue changes, especially after therapy [21]. Recently, the Response Assessment in NeuroOncology (RANO) working group-an international effort to develop new standardized response criteria for clinical trials in brain tumours-has recommended the additional use of amino acid PET imaging for brain tumour management [5]. The longest established amino acid tracer ${ }^{11} \mathrm{C}$ MET has been replaced in many neuro-oncology centres by the more convenient ${ }^{18} \mathrm{~F}$-FET, and several thousand ${ }^{18}$ F-FET PET scans have been performed in some centres in recent years [22]. The broad clinical use of ${ }^{18} \mathrm{~F}$-FET PET requires comparable quantitative parameters, but as yet, the reported cut-off values of different parameters like $\mathrm{TBR}_{\text {mean }}, \mathrm{TBR}_{\max }$, TTP, slope and $\mathrm{T}_{\mathrm{vol}}$ for tumour grading or differentiation of recurrent tumour from treatment

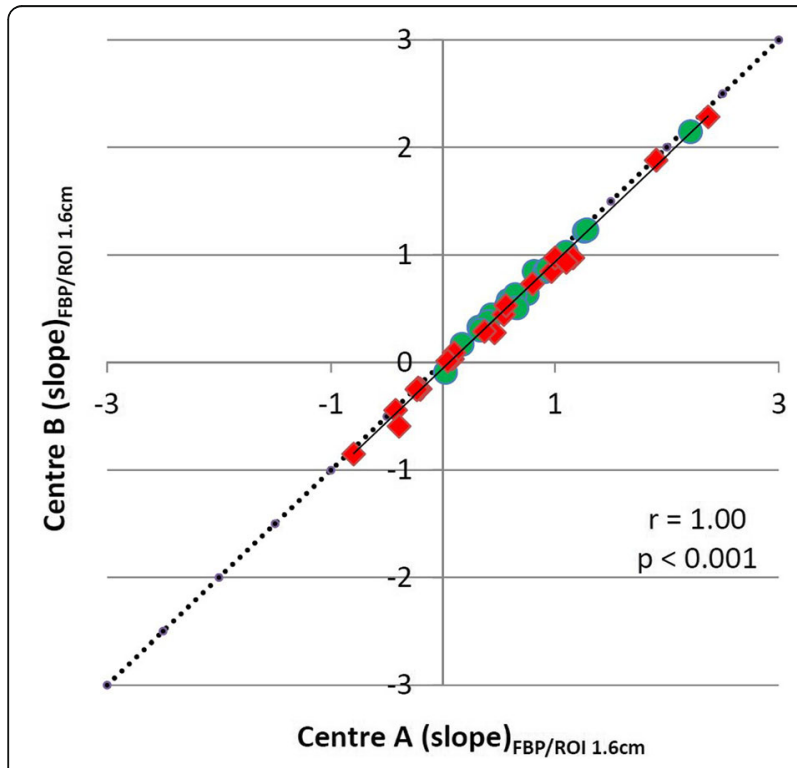

Fig. 7 Correlation of slope of the TAC of ${ }^{18} \mathrm{~F}$-FET uptake from 10 to 40 min p.i., comparing the effect of different framing in centres $A$ and $\mathrm{B}$. All data were reconstructed by FBP according to centre A and evaluated with a circular $\mathrm{ROI}$ with fixed a diameter of $1.6 \mathrm{~cm}$. There is a highly significant correlation which virtually excludes an influence of different data framing. High-grade tumours are indicated by red symbols and low-grade tumour by green symbols related changes appear to vary among different centres. It remains unclear whether diverging results are caused by the composition of the study population, differences in the technical equipment or differences in data processing or a combination thereof.

In this study, we have identified methodological differences between two centres in Germany which have a high frequency of ${ }^{18} \mathrm{~F}$-FET PET investigations and contributed to the clinical evaluation of ${ }^{18} \mathrm{~F}$-FET PET through numerous publications (for review, see $[3,5]$ ). Since in both centres, the previous publications on ${ }^{18} \mathrm{~F}$ FET PET studies were performed with the same PET scanner, differences in the technical equipment between the centres can be excluded. A comparison of data processing, however, identified methodological differences between the centres concerning the framing of PET data, data reconstruction, tumour delineation and ROI definition to evaluate tracer kinetics in the tumour. The effects of these methodological differences on quantitative parameters of FET PET were examined in a collective of 40 brain tumour patients.

One frequently used parameter for brain tumour characterization is the $\mathrm{TBR}_{\max }$ which showed approximately $15 \%$ lower values when generated with the methodology of centre A than with that of centre B. In agreement with these findings, we observed lower peak values in a phantom study when data were reconstructed according to the method of centre A. In accordance with these findings, the reported average $\mathrm{TBR}_{\max }$ values in primary brain tumours are lower in centre $A$ than in centre $\mathrm{B}$ (HGG: $\mathrm{A} / \mathrm{TBR}_{\max } 3.3 \pm 1.2$ versus $\mathrm{B} / \mathrm{TBR}_{\max }$ $3.6 \pm 1.4$, LGG: A/TBR ${ }_{\max } 2.1 \pm 1.0$ versus $\mathrm{B} / \mathrm{TBR}_{\max }$ $2.4 \pm 1.0)[23,24]$. Nevertheless, the cut-off values of the $\mathrm{TBR}_{\max }$ for differentiating between HGG and LGG in those studies were similar (cut-off: A/TBR $\max _{\max } 2.7, \mathrm{~B} /$ $\mathrm{TBR}_{\max } 2.5$ ), and the accuracy of differentiating between

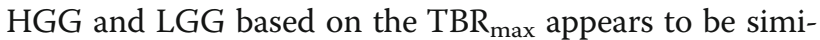
lar to both centres. For the differentiation of recurrent tumours from treatment-related changes which is a frequent clinical question, the reported cut-off value for $\mathrm{TBR}_{\max }$ was 2.0 in centre A and 2.3 in centre B which might also reflect the difference in data processing in the two centres $[12,25]$. $\mathrm{TBR}_{\text {mean }}$ values generated with the methodology of centres A and B showed a small but significant difference in the paired $t$ test $\left(\mathrm{A} / \mathrm{TBR}_{\text {mean }}\right.$ $2.20 \pm 0.41$ versus and $\left.\mathrm{B} / \mathrm{TBR}_{\text {mean }} 2.16 \pm 0.41, p<0.04\right)$, but this difference would no longer be significant if a correction for multiple testing were applied.

A major difference between both centres was observed for the definition of the biological tumour volume. The approach of centre A led to approximately $25 \%$ smaller tumour volumes than that of centre B which is of substantial importance since ${ }^{18} \mathrm{~F}$-FET PET is increasingly used when planning therapeutic interventions such as surgery 
and radiotherapy. It has to be considered that the approach of centre $\mathrm{A}$ is based on clinical experience while the approach of centre B was developed on the basis of a biopsy-controlled study which from a scientific point of view has to be regarded as more reliable [8]. On the other hand, that study is based on 52 biopsies in 31 patients only, which appears not sufficient to establish a clinical standard. Furthermore, only patients with newly diagnosed brain tumours were included, and the optimal threshold might be different for pretreated patients who can present slight unspecific tracer uptake at the primary tumour site. Therefore, further biopsy-controlled studies are needed to clarify this clinically important aspect.

The evaluation of dynamic ${ }^{18} \mathrm{~F}$-FET PET by the approach of centre A yielded shorter TTP values and lower values for the slope in the late phase of the TAC. A detailed analysis showed that the different framing procedures in centres A and B did not influence the dynamic parameters (Fig. 7) and also the ROI definition by $90 \%$ isocontour instead of TBR $>1.6$ caused only minor differences. The decisive difference was caused by the more extensive search for tumour areas with negative slope in the entire tumour volume in centre A. This approach appears to be advantageous but is technically more challenging. On the other hand, we did not detect a significant difference between the two approaches for the differentiation between HGG and LGG by ROC analysis in this group of patients.

An important reason for the differences in $\mathrm{TBR}_{\max }$ and $T_{\mathrm{vol}}$ observed in both centres is the different spatial resolution of the PET scans which is caused by the different reconstruction methods. It appears that the use of FBP in centre A is a major cause of the observed differences which is also evident from the phantom study (Fig. 2). This observation is in line with previous studies comparing the difference of FBP to OSEM on quantification of glucose metabolism which all report higher SUVs for OSEM as compared to FBP [26-28]. Since in newer PET systems, data processing is generally based on iterative reconstruction differences between centres which may become smaller in the future. In any case, the spatial resolution of PET scans should be comparable when applying threshold values for the definition of ROIs in the tumour area with FET PET. It is therefore necessary to adapt the reconstruction parameters in order to achieve a similar spatial resolution in various PET systems. Kinetic parameters are less strongly influenced by spatial resolution but are altered by the selection of specific tumour regions.

It needs to be considered that owing to the lack of molecular data for histological analysis, tumour classification was based on the WHO classification from 2007 instead of the new one from 2016, which includes molecular parameters $[18,29]$. Therefore, the AUC values obtained for tumour grading in this study might not be directly applicable to the current classification, and an influence on the comparison of the AUC values for centres A and B cannot be excluded.

\section{Conclusions}

The differences in data processing between the two centres lead to considerable differences especially for $\mathrm{TBR}_{\max }$, slope, TTP and $\mathrm{T}_{\mathrm{vol}}$. Although there was a strong linear correlation between $\mathrm{TBR}_{\max }$ and $\mathrm{T}_{\mathrm{vol}}$ values of both centres, the absolute values cannot directly be compared. Absolute $\mathrm{TBR}_{\text {mean }}$ values, by contrast, seem comparable between both centres. However, concerning the evaluation of dynamic ${ }^{18} \mathrm{~F}$-FET PET, both centres achieved comparable high accuracy to discriminate between HGG and LGG. A standardization of data processing and protocols for ${ }^{18}$ F-FET-PET is needed in order to make clinical results comparable.

\begin{abstract}
Authors' contributions
CPF, NLA, GB, BS, NJS, FMM, PB, JCT and KJL designed the study and wrote the protocol. CPF and GS performed the PET studies. CPF, ERK, NG and KJL analysed the data. CPF, NLA and KL wrote the first draft of the manuscript. All authors read and approved the final manuscript.
\end{abstract}

\section{Ethics approval and consent to participate}

All patients were investigated within a prospective study evaluating the diagnostic value ${ }^{18}$ F-FET PET in cerebral gliomas which was approved by the university ethics committee and federal authorities (study no. 2438,

University of Düsseldorf). All subjects gave prior written informed consent for their participation in the ${ }^{18}$ F-FET PET study and evaluation of their data for scientific purposes. All procedures performed in studies involving human participants were in accordance with the ethical standards of the institutional and/or national research committee and with the 1964 Helsinki declaration and its later amendments or comparable ethical standards.

Consent for publication

Not applicable.

Competing interests

The authors declare that they have no competing interests.

\section{Publisher's Note}

Springer Nature remains neutral with regard to jurisdictional claims in published maps and institutional affiliations.

\section{Author details \\ ${ }^{1}$ Institute of Neuroscience and Medicine (INM-4, INM-3), Forschungszentrum Jülich, Jülich, Germany. ${ }^{2}$ Department of Nuclear Medicine, RWTH University of Aachen, Aachen, Germany. ${ }^{3}$ Department of Nuclear Medicine, LMU Munich, Munich, Germany. ${ }^{4}$ Department of Neurosurgery, LMU Munich, Munich, Germany. ${ }^{5}$ Department of Neurology, University of Cologne, Cologne, Germany. ${ }^{6}$ Center of Integrated Oncology (ClO), Universities of Cologne and Bonn, Bonn, Germany. 'Department of Neurology, RWTH University of Aachen, Aachen, Germany. ${ }^{8}$ Jülich-Aachen Research Alliance (JARA) - Section JARA-Brain, Jülich and Aachen, Germany.}

Received: 26 June 2017 Accepted: 9 August 2017

Published online: 16 August 2017

\section{References}

1. Suchorska B, Albert NL, Tonn JC. Usefulness of PET imaging to guide treatment options in gliomas. Curr Treat Options Neurol. 2016;18:4. 
2. Galldiks N, Langen KJ, Pope WB. From the clinician's point of view-what is the status quo of positron emission tomography in patients with brain tumors? Neuro-Oncology. 2015;17:1434-44.

3. Galldiks N, Langen KJ. Applications of PET imaging of neurological tumors with radiolabeled amino acids. Q J Nucl Med Mol Imaging. 2015;59:70-82.

4. Herholz K, Langen KJ, Schiepers C, Mountz JM. Brain tumors. Semin Nucl Med. 2012;42:356-70.

5. Albert NL, Weller M, Suchorska B, Galldiks N, Soffietti R, Kim MM, et al. Response Assessment in Neuro-Oncology working group and European Association for Neuro-Oncology recommendations for the clinical use of PET imaging in gliomas. Neuro-Oncology. 2016;18:1199-208.

6. Wester HJ, Herz M, Weber W, Heiss P, Senekowitsch-Schmidtke R, Schwaiger $\mathrm{M}$, et al. Synthesis and radiopharmacology of O-(2-[18F]fluoroethyl)-Ltyrosine for tumor imaging. J Nucl Med. 1999;40:205-12.

7. Galldiks N, Langen K, Holy R, Pinkawa M, Stoffels G, Nolte K, et al. Assessment of treatment response in patients with glioblastoma using [18F]fluoroethyl-Ltyrosine PET in comparison to MRI. J Nucl Med. 2012;53:1048-57.

8. Pauleit D, Floeth F, Hamacher K, Riemenschneider MJ, Reifenberger G, Muller HW, et al. O-(2-[18F]fluoroethyl)-L-tyrosine PET combined with MRI improves the diagnostic assessment of cerebral gliomas. Brain. 2005;128:678-87.

9. Jansen NL, Suchorska B, Schwarz SB, Eigenbrod S, Lutz J, Graute V, et al. [18F]fluoroethyltyrosine-positron emission tomography-based therapy monitoring after stereotactic iodine-125 brachytherapy in patients with recurrent high-grade glioma. Mol Imaging. 2013;12:137-47.

10. Jansen NL, Suchorska B, Wenter V, Schmid-Tannwald C, Todica A, Eigenbrod $\mathrm{S}$, et al. Prognostic significance of dynamic 18F-FET PET in newly diagnosed astrocytic high-grade glioma. J Nucl Med. 2015;56:9-15.

11. Galldiks N, DunkI V, Stoffels G, Hutterer M, Rapp M, Sabel M, et al. Diagnosis of pseudoprogression in patients with glioblastoma using O-(2-[18F]fluoroethyl)L-tyrosine PET. Eur J Nucl Med Mol Imaging. 2015;42:685-95.

12. Galldiks N, Stoffels G, Filss C, Rapp M, Blau T, Tscherpel C, et al. The use of dynamic O-(2-18F-fluoroethyl)--tyrosine PET in the diagnosis of patients with progressive and recurrent glioma. Neuro-Oncology. 2015;17(9):1293-300.

13. Calcagni ML, Galli G, Giordano A, Taralli S, Anile C, Niesen A, et al. Dynamic O(2-[18F]fluoroethyl)-L-tyrosine (F-18 FET) PET for glioma grading: assessment of individual probability of malignancy. Clin Nucl Med. 2011:36:841-7.

14. Pöpperl G, Kreth FW, Mehrkens JH, Herms J, Seelos K, Koch W, et al. FET PET for the evaluation of untreated gliomas: correlation of FET uptake and uptake kinetics with tumour grading. Eur J Nucl Med Mol Imaging. 2007;34:1933-42.

15. Weckesser M, Langen KJ, Rickert CH, Kloska S, Straeter R, Hamacher K, et al. $\mathrm{O}-(2-[18 \mathrm{~F}]$ fluorethyl)-L-tyrosine PET in the clinical evaluation of primary brain tumours. Eur J Nucl Med Mol Imaging. 2005;32:422-9.

16. Jansen NL, Graute V, Armbruster L, Suchorska B, Lutz J, Eigenbrod S, et al. MRI-suspected low-grade glioma: is there a need to perform dynamic FET PET? Eur J Nucl Med Mol Imaging. 2012;39:1021-9.

17. Ceccon G, Lohmann P, Stoffels G, Judov N, Filss CP, Rapp M, et al. Dynamic $\mathrm{O}-(2-18 \mathrm{~F}$-fluoroethyl)-L-tyrosine positron emission tomography differentiates brain metastasis recurrence from radiation injury after radiotherapy. NeuroOncology. 2017;19:281-8.

18. Louis DN, Ohgaki H, Wiestler OD, Cavenee WK, Burger PC, Jouvet A, et al. The $2007 \mathrm{WHO}$ classification of tumours of the central nervous system. Acta Neuropathol. 2007;114:97-109.

19. Hamacher $\mathrm{K}$, Coenen $\mathrm{HH}$. Efficient routine production of the 18F-labelled amino acid O-2-18F fluoroethyl-L-tyrosine. Appl Radiat Isot. 2002;57:853-6.

20. Suchorska B, Jansen NL, Linn J, Kretzschmar H, Janssen $H$, Eigenbrod S, et al. Biological tumor volume in 18FET-PET before radiochemotherapy correlates with survival in GBM. Neurology. 2015;84:710-9.

21. Wen PY, Macdonald DR, Reardon DA, Cloughesy TF, Sorensen AG, Galanis E, et al. Updated response assessment criteria for high-grade gliomas: response assessment in neuro-oncology working group. J Clin Oncol. 2010;28:1963-72.

22. Langen KJ, Tonn JC, Weller M, Galldiks N. Letter to the editor: "the role of imaging in the management of progressive glioblastoma. A systematic review and evidence-based clinical practice guideline" [J Neurooncol 2014; 118:435-460]. J Neuro-Oncol. 2014;120:665-6.

23. Albert NL, Winkelmann I, Suchorska B, Wenter V, Schmid-Tannwald C, Mille E, et al. Early static (18)F-FET-PET scans have a higher accuracy for glioma grading than the standard 20-40 min scans. Eur J Nucl Med Mol Imaging. 2016:43:1105-14.

24. Rapp M, Heinzel A, Galldiks N, Stoffels G, Felsberg J, Ewelt C, et al. Diagnostic performance of 18F-FET PET in newly diagnosed cerebral lesions suggestive of glioma. J Nucl Med. 2013;54:229-35.
25. Pöpperl G, Gotz C, Rachinger W, Gildehaus FJ, Tonn JC, Tatsch K. Value of O(2-[18F]fluoroethyl)-L-tyrosine PET for the diagnosis of recurrent glioma. Eur J Nucl Med Mol Imaging. 2004;31:1464-70.

26. Etchebehere EC, Macapinlac HA, Gonen M, Humm K, Yeung HW, Akhurst T, et al. Qualitative and quantitative comparison between images obtained with filtered back projection and iterative reconstruction in prostate cancer lesions of (18)F-FDG PET. Q J Nucl Med. 2002;46:122-30.

27. Visvikis D, Cheze-LeRest C, Costa DC, Bomanji J, Gacinovic S, Ell PJ. Influence of OSEM and segmented attenuation correction in the calculation of standardised uptake values for [18F]FDG PET. Eur J Nucl Med. 2001;28:1326-35.

28. Ramos CD, Erdi YE, Gonen M, Riedel E, Yeung HW, Macapinlac HA, et al. FDG-PET standardized uptake values in normal anatomical structures using iterative reconstruction segmented attenuation correction and filtered backprojection. Eur J Nucl Med. 2001;28:155-64.

29. Louis DN, Perry A, Reifenberger G, von Deimling A, Figarella-Branger D, Cavenee WK, et al. The 2016 World Health Organization classification of tumors of the central nervous system: a summary. Acta Neuropathol. 2016; 131:803-20.

\section{Submit your manuscript to a SpringerOpen ${ }^{\circ}$ journal and benefit from:}

- Convenient online submission

- Rigorous peer review

- Open access: articles freely available online

- High visibility within the field

- Retaining the copyright to your article

Submit your next manuscript at springeropen.com 